\title{
Identification and Evaluation of Technology for Detection of Aflatoxin Contaminated Peanut
}

\author{
Chaitra C. \\ Dept. of Electronics and Communication \\ Siddaganga Institute of Technology \\ Tumkur, Karnataka, India
}

\author{
K.V. Suresh \\ Dept. of Electronics and Communication \\ Siddaganga Institute of Technology \\ Tumkur, Karnataka, India
}

\begin{abstract}
Aflatoxin belongs to a group of fungal toxins known as mycotoxins, and is widespread in agricultural products and food. Consumption of aflatoxin contaminated peanuts causes severe health problems, like immune system suppression, cancer, and may lead to death. Therefore, quality classification of peanut using an efficient non-destructive method is very essential for food grain industries. In this paper imaging techniques such as thermal imaging, fluorescence imaging and color imaging are identified and evaluated. The results show that, thermal and fluorescence imaging techniques are not suitable for detection of contaminated peanuts. Hence, an algorithm for color imaging technique is proposed as an effective alternative method to detect contaminated peanuts based on external appearance. The main objective of the proposed algorithm is to classify peanuts into good and bad, based on color feature. The captured images are first pre-processed, and database is prepared automatically. Statistical and histogram features are then extracted for classification using Feed Forward Neural Network (FFNN), and Linear Discriminant Analysis (LDA). Proposed algorithm is developed using MATLAB 7.12, and tested on several peanut samples.
\end{abstract}

\section{Keywords}

Aflatoxin, Peanut, Thermal imaging, Fluorescence imaging, Color imaging, Quality.

\section{INTRODUCTION}

Fungal contaminated food grains lead to huge economic loss. In many countries aflatoxin is a major health risk to both humans and animals, due to high level of contaminated product consumption. Therefore, to give quality food for consumer, a best nondestructive method is necessary for rapid detection of aflatoxin.

Aflatoxin is a group of fungal toxins namely B1, B2, G1, G2, M1, M2 produced by Aspergillus flavus and Aspergillus parasiticus. These toxins are secondary metabolites of aspergillus moulds. Aflatoxins are produced on various grains and nuts, e.g., peanut, corn, cotton seed, pistachio nuts, cerels, fruits, oilseeds, dried fruits, and spices in the field and during storage. These Grains when infected by Aspergillus fungus is odorless, bitter, light weight, pale yellow and powdery surface. Consumption of Aflatoxin infected grain has carcinogenic, hepatotoxic, teratogenic and mutagenic effects in humans and animals even at very small concentration $[1,2$, $3,4]$. Peanuts are the oily seeds that are most commonly consumed by humans, and are used to feed the animals. Peanuts compose of sufficient levels of mono-unsaturated fatty acids especially oleic acid. These peanuts are a good source of dietary protein composes of fine quality amino acids that are essential for growth and development [5]. But, these peanuts suffer from Aspergillus flavus fungus that produces aflatoxin. Consumption of aflatoxin contaminated peanut cause sever health problem. Hence, quality inspection of peanut plays a very important role in food grain industry.

Literature review records many papers which address the problem of aflatoxin, and methods for its detection. C. W. Hesseltine and O. Shotwell [6], proposed two new methods for rapid detection of aflatoxin in corn. First method is based on glowing greenish gold fluorescence produced under UV light by corn kernels. Second method is modification of the chromatographic mini-column method devised for corn. Various test methods, such as enzyme linked immunosarbent assay (ELISA), Electro chemical immunosensors, chromatography, Fluorescence, FT-NIR, and Spectrometry have been presented by Alejandro Espinosa-Calderon, et al., [7]. Aflatoxin is detected in corn using transmittance and reflectance spectroscopy by T. C. Pearson et al. [8]. Transmittance spectra $(500 \mathrm{~nm}$ to $950 \mathrm{~nm})$, and reflectance spectra $(550 \mathrm{~nm}$ to $1700 \mathrm{~nm})$, were analysed to distinguish aflatoxins in single corn kernel. Spectral analysis is done using discriminant analysis and partial least square regression. Hellebrand et al., [9], presented a work to detect plant disease due to fungus. Detection of infection in plant based on plant transpiration using thermal, and NIR (Near infrared) imaging. J. G. Tallada, D. T. et al., [10], evaluated the performance of NIR spectroscopy and color image processing to discriminate corn kernels infected by fungus species at different infection levels. A study was made to examine the relationship between fluorescence emission of corn kernels, and aflatoxin contamination level by H. Yao, Z. Hruska et al. [11]. Here, statistical analysis of aflatoxin Hyperspectral data is done using multiple linear regressions, multivariate analysis of variance, and discriminant analysis was implemented with the SAS program. H. Yao, Z. Hruska et al. [12], developed a system using a spectral angle mapper classification technique to classify fluorescence Hyperspectral images of single corn kernels into contaminated and healthy groups. Here peak shift of fluorescence is observed to be associated with contaminated corn. A detailed study on present status of nondestructive methods by M. Rajalakshmi and P.Subashini [13] concludes by saying multispectral images of chilli pepper may work well, and toxin detection using computational intelligence techniques shows a quite good experimental result. United States department of agriculture science (USDA) [14], started research work on development of rapid, non-destructive hyperspectral imaging methodology to measure fungal growth and aflatoxin in corn. The article published by USDA clearly states that UV detection methods do not actually detect mycotoxins. Therefore, UV methods can only be used for initial screening. Different processing 
approaches were developed and illustrated. Hong chen, Jing wang Qiaoxia yuan and pengwan [15], presented a work on classification of peanuts based on R, G, B color feature for color based classification, correlation for texture, fourier transform and inverse fourier transform for shape, and 13 harmonics description for peanut shape classification. A color based sorter for separating red and white wheat was developed by Tom pearson et al. [16]. A comparative study was done by Anil kannur et al. [17], to classify and grade bulk seed samples using artificial neural network. Three sets of features namely color, area and equidiameter are extracted for classification, and combinations of these features are tested with different artificial neural networks. A review paper by Chaoxin Zheng et al., [18], briefs how selection of features plays important role in classification. Each feature namely color, size, and texture carries useful information, using which good classification can be achieved. Atris Suyantohadi et al. [19], made a research to detect toxin in peanuts using $\mathrm{k}$ mean clustering algorithm. A method to trace the origin of peanut pods using image recognition was presented by Han Zhongzhi et al., [20].

From the literature review it is observed that, most of the work is done for corn, wheat and chilli pepper using hyperspectral and multispectral imaging. Change in color is also one of the best properties of peanut, using which quality can be assessed. Color based classification separates almost all bad peanuts. Further, size and texture based classification improves the quality of peanut. Hence, developing a rapid detection and classification algorithm based on color, size and texture is a useful work for industrial application. In this paper, thermal images and fluorescence images are analyzed, and a method is proposed for color imaging technique. The objective of the proposed method is to develop an algorithm to prepare peanut database automatically, and select suitable feature that classifies peanuts into good and bad. Linear Discriminant Analysis (LDA), and Feed Forward Neural Network (FFNN) are used for classification.

The paper is organized as follows. Section 2, contains the proposed algorithm. Section 3 discusses the experimental results, and section 4 contains the brief summary and closing remarks.

\section{PROPOSED METHOD}

The block diagram of proposed algorithm is shown in Figure 1. In image acquisition part, peanut images are captured in visible range. The captured color images are converted into binary, and then morphological operations are performed to retain the pixel information. In segmentation and object extraction part, each peanut is segmented from its background; Further, each segmented peanut is stored as a subimage to form a peanut database. The features are extracted from all the peanut images in the database, and are classified using FFNN and LDA classifier.

\subsection{Image Acquisition and Pre-processing}

Images are captured using Sony Cybershot HX200V (18 Megapixel, SLR) camera using light source and black color background. $\mathrm{R}$ component of the input image shows good difference between the foreground and background. Hence, $R$ component of the image is considered for further processing.

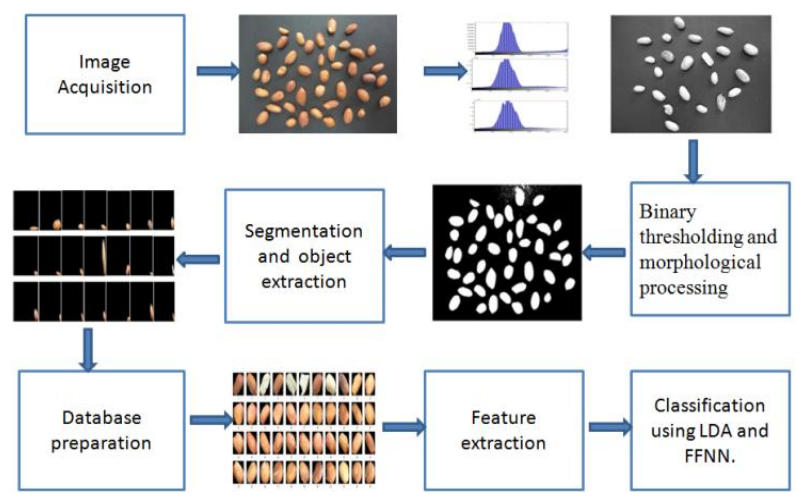

Figure 1: Block Diagram of Proposed Method

\subsection{Binary Thresholding and Morphological Processing}

The $\boldsymbol{R}$ component image is first converted into binary by thresholding,

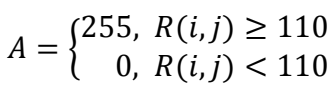

Where, $1 \leq i \leq r, 1 \leq j \leq c$ and $r \times c$ is the size of the image $R$. Binary thresholding results in dark background and bright foreground image. Two problems faced after thresholding are, loss of foreground pixel information, and some of the dust particles in the original image appear like a new object. Hence, morphological operations like hole filling, and erosion are performed on the image $A$ [8].

\subsection{Segmentation and object extraction}

Once the foreground is separated from background, it is necessary to label each object in the foreground in order to extract them separately. Each extracted objects are then stored to prepare peanut database.

Following algorithm is developed for object extraction

Consider there are $\mathrm{N}$ peanuts in the original image and $\mathrm{L}$ is the label matrix. All pixels belong to one peanut is labelled as $l_{1}$; similarly pixels of $N$ peanuts are labelled as $l_{1}, l_{2}, l_{3}, \ldots . l_{N}$.

The detailed procedure is:

Step 1: Read label $l_{1}$ and its location details from the label matrix $L$.

Step 2: Consider the pixel location obtained in step1 and go to the same location in the original image I. Retrieve R, G and $B$ values of that pixel location and store it in the same location of new sub image.

Step 3: Save new sub image in temporary database.

Step 4: Repeat step 1 to step 3 for $N$ peanuts.

From the above algorithm foreground objects are extracted from the original image. But, these images contain extra background, and that is to be removed.

\subsection{Database preparation}

An image database is prepared using automatic database preparation algorithm. This algorithm will remove dark 
background from the images, which results from the algorithm given in section 2.3.

Following are the steps of the algorithm:

Step 1: Take R, G and B component matrices of a peanut sub image.

Step 2: For R component matrix, check for all zero rows. If the row is all zero, then assign 0 to a corresponding row in a column vector, otherwise assign 1.

Step 3: Repeat Step 2 for all columns of the matrix to get a row vector.

Step 4: Repeat step 2 and step 3 for G, and B components matrices.

Step 5: Consider the column vectors from step 2. Check each row of the column vector $\mathrm{R}$ and corresponding row of the $\mathrm{G}$, and $\mathrm{B}$ component. If all three rows of column vectors are zero, then delete corresponding rows of $\mathrm{R}, \mathrm{G}$, and $\mathrm{B}$ components in the matrices.

Step 6: Consider the row vectors from step 3. Check each column of the row vector $\mathrm{R}$ and corresponding column of the $\mathrm{G}$, and $\mathrm{B}$ component. If all three columns of the row vectors are zero, then delete corresponding column of R, G, and B components in the matrices.

Step 7: R, G and B matrix values are moved to a new variable, to get color subimage of the peanut free from extra background.

Step 8: Resize all subimages to same size.

\subsection{Feature Extraction and Classification}

The feature of an object plays a key role in classification. The best feature subsets selected in this work for classification are: Percentage of red and green pixels with intensity less than 100 , and the percentage of blue pixels with the intensity less than 120. Statistical features namely, mean, median, and, standard deviation of R, G, and B components with 24 histogram features are extracted. Totally 36 features are extracted for classification. In this work FFNN and LDA are used for classification.

\section{EXPERIMENTAL RESULTS AND DISCUSSION}

Based on the physical and spectral properties of contaminated peanut, thermal images and fluorescence images are analyzed, and the results of proposed algorithm for color imaging technique are presented.

\subsection{Thermal imaging}

Temperature of the infected plants is low compared to healthy plants. Similarly, since the aflatoxin is because of fungus, a living cell, there is a chance of variation in intensity. Assuming same principle works for detection of infected peanut in the group of healthy kernels, thermal imaging is used as one of the test method in this work. Images are captured using DIR-SA-900 Series thermal imager shown in Figure 2(a); sensor is based on 25 micron pitch $384 \times 288$ uncooled micro bolometer arrays operating at room temperature. Spectral range is $8-14 \mu \mathrm{m}$.

Using the apparatus in Figure 2(a), initially peanuts were placed on black surface, and gray scale images were captured by varying contrast and brightness. After this, samples were placed on a hot object and few images were captured. Later samples were preheated for 30 seconds and placed on black surface and few images were captured. Finally, images were captured by preheating the samples followed by cooling. In all the cases it was tried to get the best image that shows appreciable difference between good and infected peanut kernels. From the Figure 2(b), (c), it is observed that, healthy peanuts are slightly brighter, compared to the contaminated peanuts. But, some of the contaminated peanuts are also appear to be brighter, and similar to the background. Also, in Figure 2(d) both healthy and contaminated peanuts are red in color. There is no useful difference in thermal image that could be made use to detect aflatoxin contaminated peanut. Thus, thermal imaging technique is not favorable for the purpose.

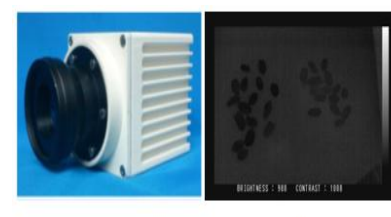

(a)

(b)

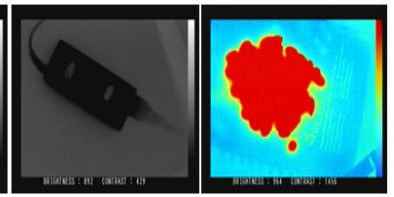

(c)

(d)

Figure 2. (a) DIR-SA-900 Series thermal imager. Peanut samples (b) placed on a black surface, (c) placed on hot surface and (d) samples are preheated for 30 seconds.

\subsection{Fluorescence imaging}

Fluorescence is a property of the fungal compounds that are produced during secondary metabolism of the aspergillus flavus fungus. Since, aflatoxin is also one of the secondary metabolite there is a chance of detecting the contaminated peanut using fluorescence imaging technique. Figure 3(a) shows the intensity of fluorescence at different wavelength for various concentration of aflatoxin.

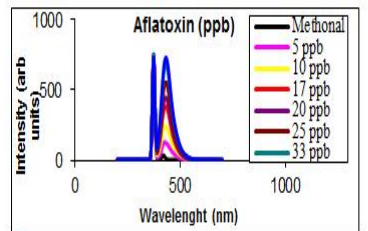

(a)

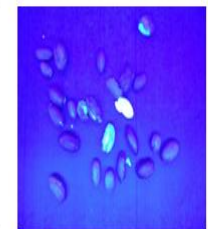

(b)

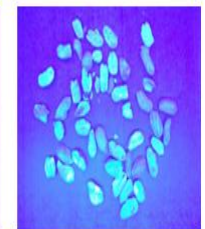

(c)
Figure 3. (a) Plot of wavelength versus fluorescence intensity, and Fluorescence image of peanut samples containing (b) both healthy and contaminates peanut, (c) only contaminated peanut

We can observe that as the concentration of aflatoxin increases, fluorescence intensity also increases. Fluorescence images are captured using sony cybershot camera with $18 \mathrm{M}$ pixel resolution under a black background, using UV LEDs of $400 \mathrm{~nm}$ wavelength as a light source. Figure 3(b), (c) are the fluorescence images. Both the images are showing appreciable difference between healthy and contaminated peanuts. But, this fluorescence is mainly because of the BGYF compounds and not aflatoxin. In Figure 3(b), we can observe that even the healthy peanuts with skin removed look like fluorescence because of the reflection of its surface, this leads to false detection. Hence, this method can only be used for initial screening. 


\subsection{Color Imaging}

Images are captured using sony cybershot camera with each image containing more than one peanut kernel. Figure 4(a) is the input color image, from the Figure 4(b) (c) (d) it is observed that histogram of $\mathrm{R}$ component shows good difference between foreground and background.

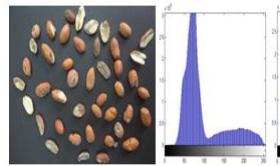

(a)

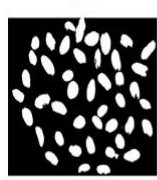

(g)
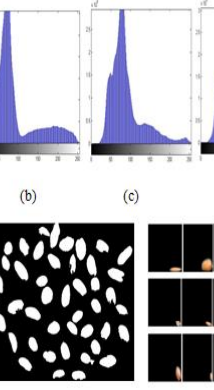

(h)

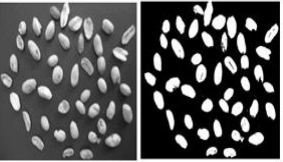

(f) Gorasiagonad rodadododana

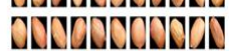

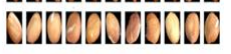

Figure 4. Colour images of peanut samples, (a) Original image, (b)(c)(d) Histogram of R,G and B components, (e)

$R$ component of image (a), (f) Image after binary

thresholding, (g) image after hole filling operation, (h) Image after erosion operation. (i) Subimages of individual peanuts extracted from original image in Figure 4(a), (j) Peanut database (resized images), after removing extra background from images in Figure 4(i)

Hence $\mathrm{R}$ component is considered in further processing. Figure 4(e) is the $R$ component of the image in Figure 4(a). Figure $4(\mathrm{f})$ is the result after binary thresholding, with the threshold value of 110. After binary thresholding some of the foreground pixels appear like background, hence it is necessary to fill the foreground region; Figure $4(\mathrm{~g})$ is the result after hole filling operation. Also, binary thresholding results in small bright dots which appear like new objects. These objects need to be removed, hence morphological erosion operation is performed using disk as a structuring element with the radius 5. But, few of the dust particles are difficult to remove by erosion operation also. Hence, to avoid these particles in the database a pixel counter is set within the object extraction algorithm. If the pixel count is less than the threshold then that object is considered as the dust particle, and it is discarded from the database. Figure 4(h) is the image after erosion operation. After pre-processing and morphological operation objects are extracted and stored as a new sub image using algorithm given in section 2.3. Figure 4(i) is the result of object extraction algorithm. Each subimage in the Figure 4(i), contains unwanted dark background. This dark background is removed using background removal algorithm given in section 2.4, and result of this algorithm is given in Figure 4(j).

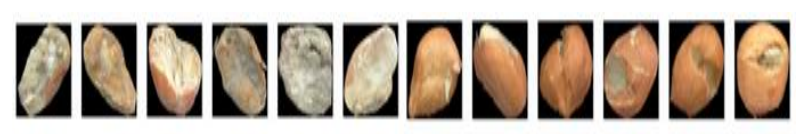

Figure 5. Bad peanut samples

After the database preparation, next step is to extract features for classification. 260 peanuts are taken for experimentation, in which 130 are good peanuts and 130 are bad peanuts. Table 1 shows classification results using LDA, and FFNN. LDA and FFNN are initially trained with 36 features. 64 samples are taken for testing.
Table 1. Classification of Red peanut samples using LDA and FFNN

\begin{tabular}{cccccc}
\hline \multirow{2}{*}{ Category } & \multicolumn{5}{c}{ Peanut sample } \\
\cline { 2 - 6 } & Input & \multicolumn{3}{c}{ Good peanuts } & \multicolumn{2}{c}{ Bad peanuts } \\
\cline { 3 - 6 } & number & Success & Failure & Success & Failure \\
\hline LDA & 32 & 32 & 0 & 31 & 1 \\
\hline FFNN & 32 & 29 & 3 & 27 & 5 \\
\hline
\end{tabular}

LDA is able to classify good peanuts with the accuracy of $100 \%$ with 0 failure number. Whereas FFNN with the accuracy of $90.62 \%$, with 3 failure number. Classification accuracy for bad peanuts reduces to $96.87 \%$ and $84.37 \%$ using LDA and FFNN respectively. Figure 5 shows the image of bad peanuts. Failure number for both good and bad peanut is mainly because, in most of the bad peanuts only a part of the endocarp is damaged, since the major part of the endocarp of such samples are similar to good ones they are misjudged as good peanuts

\section{CONCLUSION}

The thermal images look noisier and there is no appreciable difference that could be seen between aflatoxin contaminated and healthy peanut kernel. Hence, this methodology is not considered to be useful for detection of aflatoxin contaminated peanut. From the literature survey it is clear that aflatoxin does not produce fluorescence, but the other compounds associated with it will produce aflatoxin. Sometimes there is a chance that, the sample which does not fluoresce may also contain aflatoxin. Hence, fluorescence imaging does not guarantee the presence of aflatoxin. However, this method confirms the presence of fungus. Hence, it can be used for initial screening. Color imaging technique supports in detection of unhealthy peanuts based on external features caused by any fungus. But this cannot be considered has good method for aflatoxin detection. Hence, the proposed color imaging technique can detect good peanuts with the accuracy of $100 \%$, and $90.62 \%$ by LDA, and FFNN respectively. Classification accuracy for bad peanuts reduces to $96.87 \%$ and $84.37 \%$ using LDA and FFNN respectively. Some peanuts are damaged partially; hence this reduction in accuracy is mainly because of the similar features between good and bad peanuts. Thus, by using color imaging technique severely contaminated peanuts can be separated, and hence growth and spread of the aflatoxin can be avoided during storage.

\section{ACKNOWLEDGMENTS}

The authors would like to thank M/S Fowler Westrup (India) Pvt. Ltd., Bangalore for the lab facility and financial support given to carry out the work, and Dr. Chari V. Kandala, Research Agricultural Engineer, USDA, for the fluorescence plot.

\section{REFERENCES}

[1] Eduardo Micotti da Gloria, "Aflatoxin Contamination Distribution among Grain and Nuts," AflatoxinsDetection, Measurement and control, Dr. Irineo TorresPacheco (Ed.), ISBN: 978-953-307-711-6, InTech, 2011.

[2] K. Kamei, A. Wantanabe, "Aspergillus mycotoxins and their effect on the host," Medical Mycology Supplement, Taylor and Francis, 43, S95-S99, 2005. 
[3] Shami Elhaj Alssafi Bakhiet, Ahmed Altayed Altayeb Ahmed Musa, "Survey and determination of aflatoxin levels in stored peanut in sudan," Jordan Journal of Biological Sciences, Vol. 4, ISSN 1995-6673, pp. 13-20, 2011.

[4] Peter J. Cotty, Ramon Jaime-Garcia, "Influences of climate on aflatoxin producing fungi and aflatoxin contamination," International journal of food microbiology, 119, 109-115, 2007.

[5] Jocelyne Tan, Good Eating Tip of the Month, Univ. of Michigan Health System: Patient Food and Nutrition Services, February 2011.

[6] C. W. Hesseltine, O. Shotwell, "New methods for rapid detection of aflatoxin," Pure and Applied Chemistry, Vol. 35, pp. 259-266, 1973.

[7] Alejandro Espinosa-Calderon, Luis Miguel ContrerasMedina, Rafael Francisco Munoz- Huerta, Jesus Roberto Millan-Almaraz, Ramon Gerardo Guevara Gonzalez, Irineo Torres-Pacheco, "Methods for Detection and Quantification of Aflatoxins," Aflatoxins - Detection, Measurement and Control , Irineo Torres-Pacheco (Ed.), ISBN: 978-953-307-711-6, InTech, 2011.

[8] T. C. Pearson, D.T. Wicklow, E. B. Maghirang, F. Xie, F. E. Dowell,"Detecting Aflatoxin in Single Corn Kernels by Transmittance and Reflectance Spectroscopy," Transactions of the ASAE 2001, ISSN 0001-2351. Vol. 44(5):1247-1254, 2001.

[9] Hellebrand, Hans Jurgen et al.,"Plant evaluation by NIR imaging and thermal imaging".

[10] J. G. Tallada, D. T. Wicklow, T. C. Pearson, P. R. Armstrong, "Detection of fungus-infected corn kernels using near-infrared reflectance spectroscopy and color imaging," Transactions of the ASABE, Vol. 54 (3), pp1151-1158, 2011.

[11] Haibo Yao, Zuzana Hruska, Russell Kincaid, Ambrose Ononye, Robert L. Brown et al., "Correlation and classification of single kernel fluorescence Hyperspectral data with aflatoxin concentration in corn kernels inoculated with aspergillus flavus spores," Food additives and contaminants, Vol. 27, No. 5, pp-701-709, 2010.
[12] Haibo Yao, Zuzana Hruska, Russell Kincaid, Ambrose Ononye, Robert L. Brown and Thomas E. Cleveland, "Spectral angle mapper classification of fluorescence Hyperspectral image for aflatoxin contaminated corn," Hyperspectral image and signal processing: Evaluation in Remote sensing, pp. 1-4, 2010.

[13] M. Rajalakshmi, P.Subashini, "A study on nondestructive method for detecting toxin in pepper using neural networks," International journal for artificial intelligence and application, Vol. 3-Vol. 4, 2012.

[14] L. Wes Burger, ”Development of Rapid non-destructive Hyperspectral imaging methodology to measure fungal growth and aflatoxin in corn," food safety research information office, USDA. Project number: MIS-721140, 2010-2014.

[15] Hong Chen, Jing Wang, Qiaoxia Yuan, Peng Wan, "Quality Classification of peanuts based on image processing," Journal of food, Agriculture and Environment, Vol. 9 (3\&4), pp-205-209, 2011.

[16] Tom Pearson, Dan Brabee, Scott Haley, "Color image based sorter for separating red and white wheat," Sensing and Instrumentation for food quality and safety, pp. 280-288, 2008.

[17] Anil Kannur, Asha Kannur and Vijay S. Rajapurohit, "Classification And Grading Of Bulk Seeds Using Artificial Neural Networks," International Journal of Machine Intelligence, Vol. 3, pp. 62-73, 2011.

[18] Chaoxin Zheng, Da-Wen Sun and Liyun Zheng, "Recent developments and applications of image features for food quality evaluation and inspection a review," Trends in Food Science \& Technology, Vol. 17, pp. 642-655, 2006.

[19] Atris Suyantohadi and Rudiati Evi Masithoh, "Development of Machine Vision Based on Image Processing Technique to Identify Toxin Contamination In Peanuts," Australian Journal of Basic and Applied Sciences, vol. 6, pp. 135-141, 2012.

[20] Han Zhongzhi, Deng Limiao, and Yu Renshi, "Study on Origin Traceability of Peanut Pods Based on Image Recognition," International Conference on System Science, Engineering Design and Manufacturing Informatization, IEEE, vol. 2, pp. 93-96, 2011. 